\title{
DIRECT SEQUENCING OF A HLA-DRB GENE BY POLYMERASE CHAIN REACTION: SEQUENCE VARIATION IN DRw8 SPECIFICITY
}

\author{
Yoshihisa Watanabe, ${ }^{1, *}$ Katsushi TokUnaga, ${ }^{2}$ Kazumasa Matsuki, ${ }^{2}$ \\ Keiichi Омото, ${ }^{1}$ and Takeo $\mathrm{JuJ}^{2}$ \\ ${ }^{1}$ Department of Anthropology, Faculty of Science, University of Tokyo, \\ Bunkyo-ku, Tokyo 113, Japan \\ ${ }^{2}$ Blood Transfusion Service, Tokyo University Hospital, \\ Bunkyo-ku, Tokyo 113, Japan
}

\begin{abstract}
Summary The nucleotide sequence of a HLA-DRB gene with a predominant subtype of DRw8 specificity in Japanese (DR8.1) was determined with single-stranded DNA enzymatically amplified by polymerase chain reaction (PCR). The sequence differs at a single amino acid from both of the published DRw8/Dw8.1 and DRw8/Dw8.2 sequences: isoleucine ${ }^{67}$ (ATC) instead of phenylalanine ${ }^{67}$ (TTC) in DRw8/Dw8.1 and serine $^{57}$ (AGC) instead of aspartic $\operatorname{acid}^{57}$ (GAT) in DRw8/Dw8.2. On the other hand the DR8.1 and DRw8/Dw8.3 have the same amino acid sequence although one silent nucleotide substitution has occurred between the two sequences. These results indicate that Japanese DR8.1 specificity corresponds to DRw8/Dw8.3. Furthermore, an oligonucleotide probe specific for this sequence was synthesized and hybridized with 33 HLAtyped controls. This probe clearly distinguished the particular subtype from other DRw8 subtypes and specificities.
\end{abstract}

Key Words polymerase chain reaction, direct sequencing, HLA-DRw8, sequence variation

\section{INTRODUCTION}

HLA-DR antigen is one of the class II antigens of the human major histocompatibility complex (MHC). A number of serologically defined DR specificities have been known to date (WHO-HLA Nomenclature Committee, 1988). These specificities are due to the polymorphism of the DR $\beta$ chain.

The heterogeneity of the HLA-DRw8 specificity has been reported from serological, cellular, and biochemical analyses (Bétuel et al., 1984; Bouillot et al., 1988;

Received October 19, 1989; revised version received December 13, 1989; Accepted December 28, 1989.

* Present address: Department of Immunology, National Children's Medical Research Center, Setagaya-ku, Tokyo 154, Japan. 
Layrisse et al., 1984; Mickelson et al., 1983). These data suggest that there are differences in $\mathrm{DR} \beta$ chain sequences between the different DRw8 subspecificities. Four DRw8 nucleotide sequences have been reported so far. One is DRw8/Dw8.1 and predominant in Caucasians (Bell et al., 1987). DRw8/Dw8.2 is predominant in Amerindians and two sequences have been obtained from different cell lines (Jonsson et al., 1989; Gorski, 1989). The other is DRw8/Dw8.3 which is predominant in Orientals (Gorski, 1989).

Variation in number of DRB $(\beta)$ genes between the different DR specificities is also known. A single DRB gene may exist on the DRw8 carrying chromosomes, while as many as four DRB genes may occur on the DR4, DR7, and DR9 haplotypes (Böhme et al., 1985). The existence of only a single DRB gene on a DRw8bearing Japanese haplotype was also supported by the pulsed field gel electrophoresis analysis (Tokunaga et al., 1989). This situation enabled us to perform a direct sequencing of the DRw8 DRB gene with the PCR method.

In this study, we have determined the nucleotide sequence of the variable region (second exon) of a DRw8 DRB1 gene by the direct sequencing method after polymerase chain reaction and compared with those of the published DRw8 sequences.

\section{MATERIALS AND METHODS}

Source and preparation of genomic DNA. Genomic DNA was extracted from peripheral blood of a healthy Japanese (HLA A2/11, B51/w54, Cw1/w1, DR8.1/ 8.1, DRw52/w52, DQw6/w6). The DR specificity is the predominant subtype in Japanese and has been locally named as DR8.1. Homozygosity of the HLA-DR and -DQ loci has been confirmed by family study (data not shown).

In vitro DNA amplification. The oligonucleotide primers used were GLPDRB1 (5'-TTCTTCAATGGGACGGAGCG-3') and GAMPDRB1 (5'-GCCGCTGCACTGTGAAGCTCTC-3') which anneal to the sequences encoding the amino acids 17 to 23 and 87 to 94, respectively, of the DRB genes (Todd et al., 1987). For production of single-stranded DNA, in vitro DNA amplification was carried out according to Gyllensten and Erlich (1988) with minor modifications. Two $\mu \mathrm{g}$ of genomic DNA was dispensed in $100 \mu \mathrm{l}$ of PCR mixture $(50 \mathrm{~mm}$ Tris-HCl $(\mathrm{pH} 8.8$ at $25^{\circ} \mathrm{C}$ ), $10 \mathrm{mM} \mathrm{MgCl}_{2}, 10 \mathrm{~mm}$ ammonium sulfate, $1.5 \mathrm{~mm}$ of each $\mathrm{dNTP}$, and the amplification primers). Different molar amounts of the primers were applied; GLPDRB1/GAMPDRB1 ratio of 50 to $0.5 \mathrm{pmol}$ or 0.5 to $50 \mathrm{pmol}$. 2.5 units of Taq DNA polymerase (Stratagene, California) and $40 \mu 1$ of liquid paraffin were added to each sample. Then they were incubated at $95^{\circ} \mathrm{C}$ for $1 \mathrm{~min}$, at $55^{\circ} \mathrm{C}$ for $2 \mathrm{~min}$, and at $72^{\circ} \mathrm{C}$ for $1.5 \mathrm{~min}$. This cycle was repeated for 35 times, followed by an additional incubation at $72^{\circ} \mathrm{C}$ for $5 \mathrm{~min}$ to complete the extension. Aliquots $(10 \mu l)$ of the amplified DNA were electrophoresed in $4 \%$ agarose gel and amplification was verified.

For slot blotting analysis, the amplification was carried out in the same way as mentioned above except that the primers were present in equal molar amounts 
$(50 \mathrm{pmol})$ and the cycle was repeated for 25 times.

Sequencing of single-stranded DNA. Three tubes of amplified samples (total $300 \mu \mathrm{l})$ were mixed with $2 \mathrm{ml}$ of distilled $\mathrm{H}_{2} \mathrm{O}$, applied to the Centricon-30 microconcentrator, and recovered in $40 \mu 1$. The nucleotide sequences were determined by the dideoxynucleotide chain-termination method with Sequenase DNA Sequencing Kit (Toyobo, Osaka). Primers used for sequencing is one of the two amplifying primers. Seven microliters of the concentrated amplified DNA solution and 10 pmol of sequencing primer were used. The sequencing procedure was essentially according to manufacturer's recommendations. Samples were loaded onto $8 \mathrm{~m}$ urea- $8 \%$ polyacrylamide gel and electrophoresed. Gels were fixed in $10 \%(\mathrm{v} / \mathrm{v})$ acetic acid$10 \%$ (v/v) methanol, dried, and exposed to X-ray film.

Slot blotting analysis. DRB genes of 33 randomly-selected HLA-typed Japanese were amplified as mentioned above. Slot blotting and hybridization were performed as described previously except that the temperature of the hybridization and washing is $55^{\circ} \mathrm{C}$ (Watanabe et al., 1989). An oligonucleotide probe, 5'-AGGACATCCTGGAAGACAGG-3', was designed for the detection of the DR8.1 specific sequence. This probe hybridizes with the sequence encoding amino acid residues 65 to 71 of the DRB1 chain of DR8.1. Application of the enough amplified DNA to the membrane was confirmed by hybridization with $\mathrm{DR} \beta$ cDNA probe (Long et al., 1983) and positive signal was obtained in each sample.

\section{RESULTS AND DISCUSSION}

The autoradiograph of the sequencing gel is shown in Fig. 1. The nucleotide and deduced amino acid sequences of the Japanese DRw8 DRB gene (DR8.1) with those of Caucasian (Bell et al., 1987), Amerindian (Jonsson et al., 1989; Gorski, 1989), and Oriental (Gorski, 1989) DRw8 subspecificities are shown in Fig. 2. The alignment revealed that DR8.1 sequence differed from DRw8/Dw8.1 and DRw8/ Dw8.2 at one amino acid position. At the 57th amino acid position, DRw8/Dw8.2 has aspartic acid while the others have serine. At the 67th amino acid position, DR8.1 has isoleucine while the others have phenylalanine. Compared to DRw8/ Dw8.3, the DR8.1 has the same amino acid sequence in spite of a single silent substitution. The substitution occurred at the 108th position (Fig. 2), which is the third character of the codon (GAG in DR8.1 has changed to GAA in DRw8/Dw8.3) and both codons encode glutamic acid. A similar situation also occurred between two DRw8/Dw8.2 sequences at the 270th position (Fig. 2). ACG in the cell line SPL has substituted by ACA in the cell line OLGA, and both are for tyrosine. These results indicate that Japanese DR8.1 corresponds to DRw8/Dw8.3. No discrepancy was observed at the other positions.

To confirm this result, we analyzed genomic DNAs from 33 HLA-typed individuals by slot blotting with an oligonucleotide probe specific for DR8.1 sequence. Figure 3 shows the representative blot. This probe clearly identified the DR8.1 sequence (Fig. 3, individual 6 and 8 ). Interestingly, this probe also hybridizes 


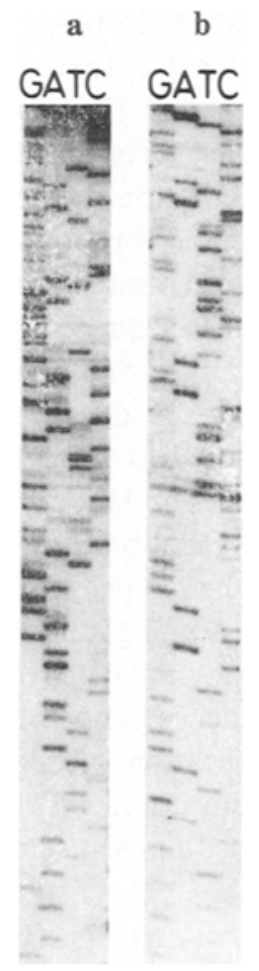

Fig. 1. Direct sequencing of the single-stranded DNA amplified by PCR. (a) Singlestranded DNA of DR8.1 was generated with $0.5 \mathrm{pmol}$ of primer GLPDRB1 and 50 pmol of primer GAMPDRB1, and sequenced with GLPDRB1. (b) Singlestranded DNA of DR8.1 was generated with 50 pmol of primer GLPDRB1 and 0.5 pmol of primer GAMPDRB1, and sequenced with GAMPDRB1.

with the sequence of DRw12 specificity (Fig. 3, individual 10). The sequence of the DRw12 DRBl gene has been reported by Navarrete et al. (1989), which shares the same sequence with the DR8.1 in this region.

In Japanese, there are two other subspecificities of DRw8 called locally as DR8.0 and DR8.2. According to the nomenclature suggested in the 9th International Histocompatibility Workshop (Grosse-Wilde et al., 1984), DR8.0 may correspond to DRw8/Dw8.1. The DR8.1 specific oligonucleotide probe detected neither DR8.0 nor DR8.2 specificities (Fig. 3). Furthermore, in a preliminary slot blotting experiment using a DRw8/Dw8.1 specific oligonucleotide probe, which anneals to the sequence encoding the amino acids 55 to 60 (Watanabe et al., 1989), the probe hybridized with DR8.0 and DR8.1 sequences, but not with DR8.2 sequence (unpublished data). Although no other information has been available about the DR8.0 and DR8.2 sequences in Japanese, it is likely that these genes also have unique sequences. 


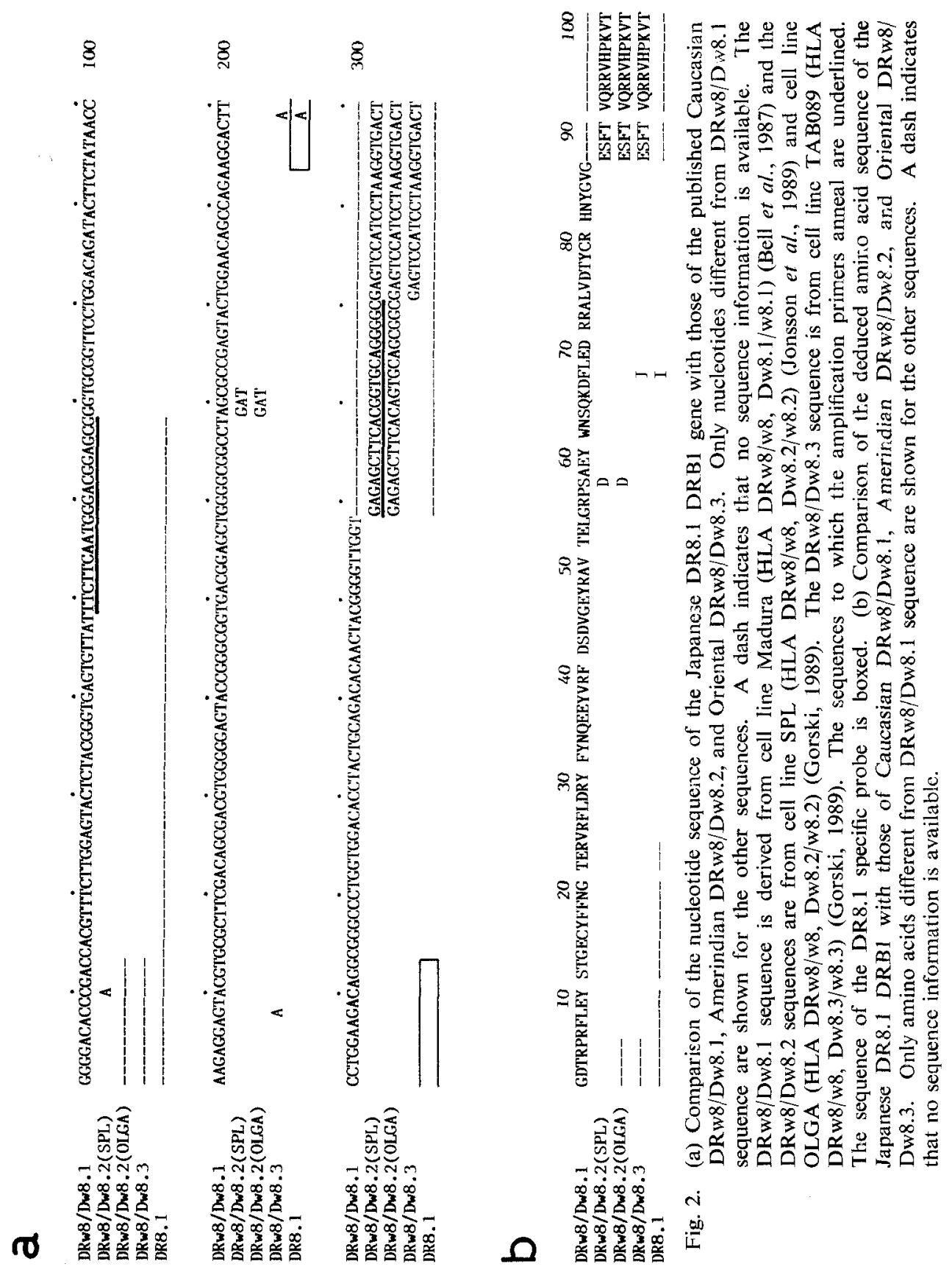

Vol. 35, No. 2, 1990 


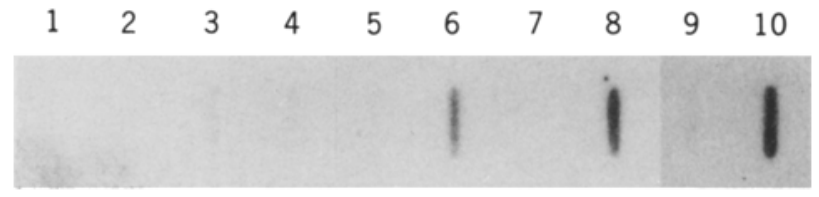

Fig. 3. Representative slot blotting analysis of HLA-typed normal controls. A specific segment of the HLA-DRB gene was amplified by the polymerase chain reaction method and hybridized with a DR8.1 specific oligonucleotide probe. The DR type of each individual is as follows: $1, \mathrm{DR} 8.2 /$ w11; $2, \mathrm{DR} 2 / 8.0 ; 3, \mathrm{DR} 9 /-; 4$, DR4/9; 5, DR2/9; 6, DR2/8.1; 7, DRw14/-; 8, DR8.1/9; 9, DRw13/w14; 10, DRw12/-.

For understanding the evolution of the DRB genes and the relationships between their structures and functions, the nucleotide sequences of other DR alleles are to be determined.

Note added in proof: To confirm the nucleotide substitution between DR8.1 and DRw8/Dw8.3 at the 108th position, genomic DNAs from other DR8.1 positive individuals were analyzed by PCR-RFLP method using RsaI. DR8.1 has the enzyme recognition site, while the site is lost in the DRw8/Dw8.3 sequence (Fig. 2). All of the six DR8.1 individuals including three individuals bearing A2-Bw46-DRw8 haplotype, which is the same haplotype as that of cell line TAB089 (DRw8/Dw8.3), had RsaI site at the position (data not shown). The result indicates that most, if not all, of Japanese DR8.1 DRB1 genes have the same sequence as we have determined in this study.

Acknowledgments We are grateful to Mr. Masami Itoh for his excellent technical assistance and to Dr. Shoji Kuwata for his helpful discussion.

This investigation was supported in part by grants from the Ministry of Education, Science and Culture of Japan.

\section{REFERENCES}

Bell, J.I., Denney, D., Foster, L., Belt, T., Todd, J.A. and McDevitt, H.O. 1987. Allelic variation in the DR subregion of the human major histocompatibility complex. Proc. Natl. Acad. Sci. U.S.A. 84: 6234-6238.

Bétuel, H., Gebuhrer, L., Schreuder, G.M.T., Layrisse, Z., Arnaiz-Villena, A. and Goldman, S.F. 1984. HLA-DRw8 antigen report. In Histocompatibility Testing 1984, Albert, E.D., Baur, M.P. and Mayr, W.R., eds., Springer-Verlag, Berlin, Heidelberg, pp. 198-200.

Böhme, J., Andersson, M., Andersson, G., Moeller, E., Peterson, P.A. and Rask, L. 1985. HLA$\mathrm{DR} \beta$ genes vary in number between different $\mathrm{DR}$ specificities, whereas the number of $\mathrm{DQ} \beta$ genes is constant. J. Immunol. $135: 2149-2155$.

Bouillot, M., Choppin, J., Sterkers, G., Freidel, C., Gebuhrer, L., Betuel, H. and Levy, P. 1988. The two-dimensional migration pattern of the single HLA-DR beta chain expressed in DRw8 haplotypes is not fully predictive of its activity in antigen presentation. Immunogenetics 28 : $46-49$.

Gorski, J. 1989. The HLA-DRw8 lineage was generated by a deletion in the DR B region fol- 
lowed by first domain diversification. J. Immunol. 142: 4041-4045.

Grosse-Wilde, H., Doxiadis, I. and Brandt, H. 1984. Definition of HLA-D with HTC. In Histocompatibility Testing 1984, Albert, E.D., Baur, M.P. and Mayr, W.R., eds., Springer-Verlag, Berlin, Heidelberg, pp. 249-264.

Gyllensten, U.B. and Erlich, H.A. 1988. Generation of single-stranded DNA by the polymerase chain reaction and its application to direct sequencing of the HLA-DQA locus. Proc. Natl. Acad. Sci. U.S.A. 85: 7652-7656.

Jonsson, A.-K., Andersson, L. and Rask, L. 1989. A cellular and functional split in the DRw8 haplotype is due to a single amino acid replacement (DR $\beta$ ser57-asp57). Immunogenetics 29: 308-316.

Layrisse, Z., Carreño, B., Svejgaard, A., Platz, P., Ryder, L.P. and Kristensen, T. 1984. First level testing of Dw8 HTC. In Histocompatibility Testing 1984, Albert, E.D., Baur, M.P. and Mayr, W.R., eds., Springer-Verlag, Berlin, Heiderlberg, pp. 278-281.

Long, E.O., Wake, C.T., Gorski, J. and Mach, B. 1983. Complete sequence of an HLA-DR $\beta$ chain deduced from a cDNA clone and identification of multiple non-allelic DR $\beta$ chain genes. EMBO J. 2: 389-394.

Mickelson, E.M., Nisperos, B., Layrisse, Z., Kim, S.J., Thomas, E.D. and Hansen, J.A. 1983. Analysis of the HLA-DRw8 haplotype: recognition by HTC typing of three distinct antigen complexes in Caucasians, native Americans, and Orientals. Immunogenetics 17: 399-410.

Navarrete, C., Seki, T., Miranda, A., Winchester, R. and Gregersen, P.K. 1989. DNA sequence analysis of the HLA-DRw12 allele. Hum. Immunol. 25: 51-58.

Todd, J.A., Bell, J.I. and McDevitt, H.O. 1987. HLA-DQ $\beta$ gene contributes to susceptibility and resistance to insulin-dependent diabetes mellitus. Nature 329: 599-604.

Tokunaga, K., Kay, P.H., Christiansen, F.T., Saueracker, G. and Dawkins, R.L. 1989. Comparative mapping of the human major histocompatibility complex in different racial groups by pulsed field gel electrophoresis. Hum. Immunol. 26: 91-97.

Watanabe, Y., Tokunaga, K., Matsuki, K., Takeuchi, F., Matsuta, K., Maeda, H., Omoto, K. and Juji, T. 1989. Putative amino acid sequence of HLA-DRB chain contributing to rheumatoid arthritis susceptibility. J. Exp. Med. 169: 2263-2268.

WHO-HLA Nomenclature Committee. 1988. Nomenclature for factors of the HLA system, 1987. Vox Sang. 55: 119-126. 